\title{
Kull ALE: I. Unstructured Mesh Advection, Interface Capturing, and Multiphase 2T RHD with Material Interfaces
}

\author{
P. Anninos
}

This article was submitted to High Energy Astrophysics Division of American Astronomical Society, April Convention 2002

Albuquerque, NM, April 20 - 23, 2002

U.S. Department of Energy

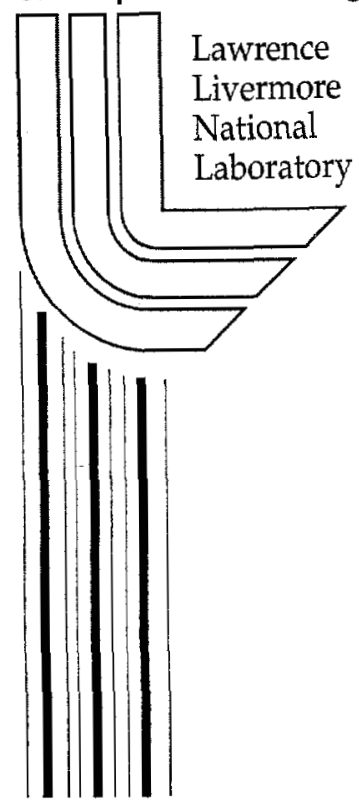

February 11, 2002 


\section{DISCLAIMER}

This document was prepared as an account of work sponsored by an agency of the United States Government. Neither the United States Government nor the University of California nor any of their employees, makes any warranty, express or implied, or assumes any legal liability or responsibility for the accuracy, completeness, or usefulness of any information, apparatus, product, or process disclosed, or represents that its use would not infringe privately owned rights. Reference herein to any specific commercial product, process, or service by trade name, trademark, manufacturer, or otherwise, does not necessarily constitute or imply its endorsement, recommendation, or favoring by the United States Government or the University of California. The views and opinions of authors expressed herein do not necessarily state or reflect those of the United States Government or the University of California, and shall not be used for advertising or product endorsement purposes.

This is a preprint of a paper intended for publication in a journal or proceedings. Since changes may be made before publication, this preprint is made available with the understanding that it will not be cited or reproduced without the permission of the author.

This work was performed under the auspices of the United States Department of Energy by the University of California, Lawrence Livermore National Laboratory under contract No. W-7405-Eng-48.

This report has been reproduced directly from the best available copy.

Available electronically at http://www.doc.gov/bridge

Available for a processing fee to U.S. Department of Energy And its contractors in paper from

U.S. Department of Energy

Office of Scientific and Technical Information

$$
\text { P.O. Box } 62
$$

Oak Ridge, TN 37831-0062

Telephone: (865) 576-8401

Facsimile: (865) 576-5728

E-mail: reports@adonis.osti.gov

Available for the sale to the public from

U.S. Department of Commerce

National Technical Information Service

5285 Port Royal Road

Springfield, VA 22161

Telephone: (800) 553-6847

Facsimile: (703) 605-6900

E-mail: orders@ntis.fedworld.gov

Online ordering: http://www.ntis.gov/ordering.htm

\section{OR}

Lawrence Livermore National Laboratory

Technical Information Department's Digital Library

http://www.llnl.gov/tid/Library.html 


\title{
Kull ALE: \\ I. Unstructured mesh advection, interface capturing, and multiphase 2T RHD with material interfaces
}

\author{
Peter Anninos \\ University of California, \\ Lawrence Livermore National Laboratory, Livermore CA 94550
}

January 28,2002

\begin{abstract}
Several advection algorithms are presented within the remap framework for unstructured mesh ALE codes. The methods discussed include a generic advection scheme based on a finite volume approach, and three groups of algorithms for the treatment of material boundary interfaces. The interface capturing algorithms belong to the Volume of Fluid (VoF) class of methods to approximate material interfaces from the local fractional volume of fluid distribution in arbitrary unstructured polyhedral meshes appropriate for the Kull code. Also presented are several schemes for extending single material radiation diffusion solvers to account for multi-material interfaces.
\end{abstract}

\section{Basic Advection}

The general mass, momentum and energy continuity equations are written in a moving mesh framework as

$$
\begin{aligned}
\frac{\partial \rho}{\partial t} & =-\rho \nabla_{i} v^{i}-\left(v^{i}-v_{g}^{i}\right) \nabla_{i} \rho, \\
\frac{\partial\left(\rho v^{k}\right)}{\partial t} & =-\nabla_{k} P-\rho v^{k} \nabla_{i} v^{i}-\left(v^{i}-v_{g}^{i}\right) \nabla_{i}\left(\rho v^{k}\right), \\
\frac{\partial(\rho \epsilon)}{\partial t} & =-P \nabla_{i} v^{i}-\rho \epsilon \nabla_{i} v^{i}-\left(v^{i}-v_{g}^{i}\right) \nabla_{i}(\rho \epsilon),
\end{aligned}
$$

where an implicit summation convention is assumed over repeated covariant and contravariant indices ( $i$ in this case). $\rho$ is the fluid density, $P$ the pressure, $v^{k}$ the velocity components, $\epsilon$ the specific energy, and $v_{g}^{k}$ the grid velocity components. A staggered mesh scheme is utilized to solve the conservation equations, with zone-centered scalar variables $(\rho, P, \epsilon)$ and node-centered velocities and momenta. The Lagrangian formulation is recovered simply by setting $v_{g}^{i}=v^{i}$ and $\partial / \partial t \rightarrow d / d t$; the Eulerian limit by definition assumes the mesh is fixed in time so $v_{g}^{i}=0$.

Decomposing equations (1.1) - (1.3) into their respective Lagrangian and remap parts by incorporating the convective derivatives into the total time derivative on the left-hand-side yields

$$
\frac{d f}{d t}=S\left(f, v^{k}, P\right)+v_{g}^{i} \nabla_{i} f
$$


where $f$ represents any of the density, momentum or energy density fields. This set of equations is solved in a split manner with explicit Lagrangian $d f / d t=S$, and remap $d f / d t=v_{g}^{i} \nabla_{i} f$ parts which solve the dynamical and kinematical elements respectively. It should be noted that due to the nonphysical nature of the mesh velocity $v_{g}^{i}$, the time step in the remap cycle is not linked to the real physical time step in the Lagrange cycle, but is in fact an arbitrary unit defined by the desired absolute nodal displacement, and can thus be normalized to unity. Any number of physical time steps can be performed between remap cycles, and the frequency of remapping is generally determined by the quality of the evolving mesh. Expanding the second term of (1.4) and using an operator splitting procedure in the remap cycle allows for a fully conservative method of solution by sequentially solving

$$
\frac{d f}{d t}=\nabla_{i}\left(v_{g}^{i} f\right)
$$

followed by

$$
\frac{d f}{d t}=-f \nabla_{i}\left(v_{g}^{i}\right)=-\frac{f}{V} \frac{d V}{d t}
$$

By identifying the velocity divergence term with the relative rate of change in the local cell volume $V$, equation (1.6) has the discrete solution $f^{n+1}=f^{n+1 / 2} V^{n} / V^{n+1}$, where $f^{n+1}$ is the full time advanced solution of (1.5) and (1.6) together, and $f^{n+1 / 2}$ is the solution of (1.5) which is discussed below and in section $\S 2$ devoted to interface capturing algorithms. Equations (1.1) - (1.6) are equivalent to solving

$$
\begin{aligned}
\frac{\partial(\rho J)}{\partial t} & =-J \nabla_{i}\left(\rho\left(v^{i}-v_{g}^{i}\right)\right) \\
\frac{\partial\left(\rho v^{k} J\right)}{\partial t} & =-J \nabla_{i}\left(\rho v^{k}\left(v^{i}-v_{g}^{i}\right)\right)-J \nabla_{k} P \\
\frac{\partial(\rho \epsilon J)}{\partial t} & =-J \nabla_{i}\left(\rho \epsilon\left(v^{i}-v_{g}^{i}\right)\right)-J P \nabla_{i} v^{i}
\end{aligned}
$$

where $J \equiv V^{n+1} / V^{n} \equiv \partial\left(x^{n+1}, y^{n+1}, z^{n+1}\right) / \partial\left(x^{n}, y^{n}, z^{n}\right)$ is the Jacobian of the coordinate transformation satisfying the conservation equation

$$
\frac{\partial J}{\partial t}=J \nabla_{i} v_{g}^{i}
$$

\subsection{Advection of zone-centered fields}

When interface capturing is not needed, equation (1.5) is solved for the zone-centered quantities $(f \equiv \rho$ or $\rho \epsilon)$ in a straight-forward manner using a single step, first order in time marching scheme. A finite volume approach is used to compute the right-hand-side of (1.5) by converting the infinitesimal volume integrals to discrete area summations over the faces in each zone

$$
\nabla_{i}\left(v_{g}^{i} f\right)=\frac{1}{V_{Z}} \int_{d V} \nabla_{i}\left(f v_{g}^{i}\right) d V=-\frac{1}{V_{Z}} \oint\left(f v_{g}^{i}\right) d A_{i}=-\frac{1}{V_{Z}} \sum_{Z F}\left(f v_{g}^{i}\right)_{Z F}\left(A_{i}\right)_{Z F}
$$

where $V_{Z}$ is the zone volume, $\left(f v_{g}^{i}\right)_{Z F}$ is the extrapolated value of the flux from the donor cell center to the cell faces, and $\left(A_{i}\right)_{Z F}$ is the face area vector normal. The area normals of each face are defined as the sum of the inward-pointing (towards the cell center) face-faceted area vectors 
of each of the tetrahedral side elements (defined by two nodes, a face center and the zone center) that make up the face $\left(A_{i}\right)_{Z F}=\sum_{Z S}\left(A_{i}\right)_{Z S} .\left(v_{g}^{i}\right)_{Z F}$ are the mesh velocity components at the face center, defined as the average of the velocities on the nodes making up the faces. $f_{Z F}$ is the first order extrapolated zone-centered field computed by the Taylor's expansion

$$
f_{Z F}=f_{Z}+\left(\nabla_{i} f\right)_{Z}^{L}\left(r^{i}-r_{Z}^{i}\right)
$$

from the donor cell center $r_{Z}^{i}$ to either the face center $r^{i}=r_{Z F}^{i}$ or the advective control volume center $r^{i}=r_{Z F}^{i}+(\Delta t / 2)\left(v_{g}^{i}\right)_{Z F}$.

$\left(\nabla_{i} f\right)_{Z}^{L}$ in (1.12) is the zone-centered gradient limited to force monotonicity in the extrapolated field variable. This is achieved by identifying three unique control volumes (assigned as upstream $\nabla_{U} f$, downstream $\nabla_{D} f$, or average $\nabla_{A} f$ gradient operators) by the sign of the inner product of $\vec{A} \cdot \vec{V} \equiv\left(A_{i}\right)^{S F}\left(v_{g, N 1}^{i}+v_{g, N 2}^{i}\right) / 2$, where $\left(A_{i}\right)^{S F}$ are the components of the face-faceted area vector of the side unit, and $v_{g, N 1}^{i}$ and $v_{g, N 2}^{i}$ are the velocity vectors at the two nodes comprising the unique edge length of the side. Each of the gradient operators are computed in a similar manner as (1.11) except replacing the sum over cell faces by sums over the side faces

$$
\nabla_{[U D A]}^{i}=-\frac{\sum_{S} M_{[U D A]}^{S}\left[f_{F S}\left(A^{i}\right)_{F S}+f_{S} \sum_{\alpha}\left(A^{i}\right)_{\alpha S}\right]}{\sum_{S} M_{[U D A]}^{S}\left(V_{S}\right)}
$$

where $M_{[U D A]}^{S}$ is a masking function set to either zero or unity depending on the sign of $\vec{A} \cdot \vec{V}$ identifying the side as contributing to the upstream, downstream or average gradients. A particular side is associated with the upstream (downstream) gradient if $\vec{A} \cdot \vec{V}>\delta(<-\delta)$, where $\delta \ll 1$. The average gradient is a composite sum of the upstream and downstream geometries, and thus includes all the cell faces. The effective extrapolated field variables (to the face centers of the side elements) can be computed as a geometrically weighted average of the zone-centered fields. For example, the simplest equidistant weighting scheme approximates $f_{F S}=\left(f_{Z D}+f_{Z+}\right) / 2$ and $f_{S}=c_{1} f_{Z D}+c_{2} f_{Z+}$, where $f_{Z D}$ is the donor cell centered field, $f_{Z+}$ is the field value at the center of the opposite zone sharing the face, and $\left(c_{1}, c_{2}\right)=(1,2 / 3)$ in $3 \mathrm{D}$ and $\left(c_{1}, c_{2}\right)=(1 / 2,1 / 2)$ in $2 \mathrm{D}$. $\left(A^{i}\right)_{F S}$ in $(1.13)$ is the area vector of the face-faceted side surface, and $\left(A^{i}\right)_{\alpha S}$ are the area vectors of the remaining $\alpha$ (equal to two in $2 \mathrm{D}$ and three in $3 \mathrm{D}$ ) surfaces of the tetrahedral side $S$. In $3 \mathrm{D}$ these area vectors are

$$
\begin{aligned}
\sum_{\alpha}(A)_{\alpha S} & =\frac{1}{2}\left(\vec{r}_{N 2}-\vec{r}_{Z}\right) \times\left(\vec{r}_{N 1}-\vec{r}_{Z}\right) \\
& +\frac{1}{2}\left(\vec{r}_{Z F}-\vec{r}_{Z}\right) \times\left(\vec{r}_{N 2}-\vec{r}_{Z}\right)+\frac{1}{2}\left(\vec{r}_{N 1}-\vec{r}_{Z}\right) \times\left(\vec{r}_{Z F}-\vec{r}_{Z}\right)
\end{aligned}
$$

where $\vec{r}_{Z}, \vec{r}_{Z F}, \vec{r}_{N 1}$, and $\vec{r}_{N 2}$ are the vector coordinates of the zone center, face center, and the two nodes of the side element.

To enforce monotonicity, the actual reconstructed gradient $(\nabla f)_{Z}^{L}$ is set to zero if the inner product of any combination of the three gradient operators is less than a predetermined small number (i.e., $(\nabla f)_{Z}^{L}=0$ if $\nabla_{U}^{i} f \nabla_{i}^{A} f<\delta$, or $\nabla_{U}^{i} f \nabla_{i}^{D} f<\delta$, or $\nabla_{A}^{i} f \nabla_{i}^{D} f<\delta$, with $\delta \ll 1$ ). The final gradient is further limited to various degrees of sharpness by defining the normalized scalar

$$
\theta=\frac{\max \left[0, \nabla_{i}^{U} f \nabla_{A}^{i} f\right]}{\max \left[0, \nabla_{i}^{D} f \nabla_{A}^{i} f\right]+\delta}
$$


and applying either of the minmod, van Leer, or superbee limiters

$$
\begin{aligned}
\phi & =\max [0, \min (1, \theta)] \\
\phi & =\frac{|\theta|+\theta}{1+|\theta|}, \\
\phi & =\max [\min (1,2 \theta), \min (2, \theta)]
\end{aligned}
$$

to get the final expression for the zone-centered limited gradient

$$
(\nabla f)_{Z}^{L}=\max \left[0, \nabla_{i}^{D} f \nabla_{A}^{i} f\right] \times \max [0, \phi] \times \frac{\nabla_{A} f}{\left|\nabla_{A} f\right|^{2}+\delta} .
$$

A different, though somewhat more restrictive and costly, method of applying a gradient limiter on an unstructured mesh is to modify the magnitude of the average gradient operator with some parameterized function $(g)$ of the maximum, $\nabla_{\max } \equiv \max \left(\left|\nabla_{U} f\right|,\left|\nabla_{A} f\right|,\left|\nabla_{D} f\right|\right)$, and minimum, $\nabla_{\min } \equiv \min \left(\left|\nabla_{U} f\right|,\left|\nabla_{A} f\right|,\left|\nabla_{D} f\right|\right)$, of the three masked gradient magnitudes

$$
\left(\nabla_{i} f\right)_{Z}^{L}=\alpha g\left[\beta \nabla_{\max },(1-\beta) \nabla_{\min }\right] \times \frac{\nabla_{A}^{i} f}{\left|\nabla_{A}^{i} f\right|+\delta},
$$

where $\beta$ is a steepness parameter bounded by $0 \leq \beta \leq 1$, and $\alpha$ is a coefficient to enforce monotonicity in the extrapolated field $f_{Z F}$ :

$$
\alpha=\min \left(1, \max \left(0, \min \left(\alpha_{1}, \alpha_{2}\right)\right)\right)
$$

where

$$
\alpha_{1}=\frac{\max \left(0, f_{Z+}-f_{Z}\right)}{f_{Z F}-f_{Z}+\delta}
$$

if $f_{Z F}=f_{Z}+\left(\nabla_{i} f\right)_{Z}^{L}\left(r_{Z F}^{i}-r_{Z}^{i}\right) / \alpha>\max \left(f_{Z}, f_{Z+}\right)$ or $\alpha_{1}=1$ otherwise, and

$$
\alpha_{2}=\frac{\min \left(0, f_{Z+}-f_{Z}\right)}{f_{Z F}-f_{Z}+\delta}
$$

if $f_{Z F}<\min \left(f_{Z}, f_{Z+}\right)$ or $\alpha_{2}=1$ otherwise. The $\min / \max$ operations in (1.21) - (1.22) are performed over each of the faces in the zones, and $\alpha$ is chosen as the smallest value needed for strict monotonicity across all the faces.

\subsection{Advection of node-centered fields}

The node-centered momentum equation, which assumes a single velocity for all materials, is solved in a similar manner as described above for the zone-centered variables. The only difference being the use of nodal (as opposed to zonal) control volumes composed of all the sub-zonal corner volume elements attached to the specified node and collectively connecting the neighboring zone centers. An effective node-centered mass density $\rho_{N}$ is also constructed as a corner-volume weighted average of the corner-centered density fields, and evolved consistently with the momentum equation. In particular, defining the nodal density as the volume average over nodally attached corners with 
$\rho_{N}=\sum_{C} V_{C} \rho_{C} / \sum_{C} V_{C}$ at the beginning of the evolution cycle, an evolution equation is solved for $\rho_{N}$ by constructing a total density flux over all materials $m$

$$
\frac{d \rho_{N}}{d t}=\nabla_{i}\left[\sum_{m}\left(\rho_{N} v_{g}^{i}\right)_{m}\right] .
$$

The flux for each material in (1.24) is first evaluated on the zone faces from the zone-centered density evolution step (see $\S 1.1$ ), then averaged (with volume weighting) to the face centers of the nodal control volume boundaries. The fluid velocity components $\left(v^{k}\right)$ are then updated to the $(n+1)$ st time level by

$$
\left(v^{k}\right)^{n+1}=\left[\left(s^{k}\right)^{n}+\Delta t\left(\frac{d s^{k}}{d t}\right)^{n}\right] \frac{1}{\rho_{N}^{n+1}},
$$

where $\left(s^{k}\right)^{n}=\rho_{N}^{n}\left(v^{k}\right)^{n}$ is the momentum vector at the current time level $n$, and $\rho_{N}^{n+1}$ is the solution of (1.24) at the time advanced level $n+1 . d s^{k} / d t$ solves (1.5) in the form

$$
\begin{aligned}
\frac{d s^{k}}{d t} & =\nabla_{i}\left(s^{k} v_{g}^{i}\right)=\nabla_{i}\left[v^{k}\left(\sum_{m}\left(\rho v_{g}^{i}\right)_{m}\right)\right] \\
& =-\frac{1}{V_{N}} \sum_{N F}\left[v_{N F}^{k}\left(\sum_{m}\left(\rho v_{g}^{i}\right)_{m}\right)_{N F}\right]\left(A_{i}\right)_{N F},
\end{aligned}
$$

where $\left(\sum_{m}\left(\rho v_{g}^{i}\right)_{m}\right)_{N F}$ represents the cumulative (over fluids) zone-centered mass flux averaged to the nodal control volume face centers, and

$$
v_{N F}^{k}=v_{N}^{k}+\left(\nabla_{i} v^{k}\right)_{N}^{L}\left(r^{i}-r_{N}^{i}\right)
$$

is a first order extrapolation of the node-centered velocity $v_{N}^{k}$ components to either the nodal control volume faces $r^{i}=r_{N F}^{i}$ or the advective control volume center $r^{i}=r_{N F}^{i}+(\Delta t / 4)\left(v_{g, N 1}^{i}+v_{g, N 2}^{i}\right)$ using a node-centered limited gradient similar to that described above for zone-centered fields.

\section{Unsplit Interface Capturing}

Multi-material interfaces in nonreactive incompressible fluids are advected in an Eulerian reference frame according to

$$
\sum_{m=1}^{M}\left\{\frac{\partial F^{[m]}}{\partial t}+\nabla_{i}\left(v_{f}^{i} F^{[m]}\right)\right\}=0
$$

where $M$ is the total number of materials, $v_{f}^{i}$ is the fluid velocity, and $F^{[m]}$ is the volume fraction occupied by the $m$ th fluid material and defined by a step function: $F^{[m]}=0$ if the cell is empty of material $m, F^{[m]}=1$ if the cell is completely filled with material $m$, and $0<F^{[m]} \equiv V^{[m]} / V_{Z}<1$ in cells containing an interface boundary, where $V_{Z}=\sum_{m=1}^{M} V^{[m]}$ is the total cell volume. Equation (2.29) is associated with the first conservative remap step with $v_{f}^{i} \rightarrow-v_{g}^{i}$ and $f \rightarrow F^{[m]}$, and the methods described below to solve (2.29) are also relevant for (1.5).

Equation (2.29) is solved only in those cells containing an interface boundary and satisfying $F_{c}<\max \left(F^{[m]}\right)<1-F_{c}$, where the lower bound cut-off volume fraction $F_{c}$ is set to a small 
number, typically $<10^{-6}$. An unsplit finite volume approach is used to solve $(2.29)$ for each material $F^{[m]}$ at time level $n+1$

$$
\left(F^{[m]}\right)^{n+1}=\left(F^{[m]}\right)^{n}+\frac{1}{V_{Z}} \sum F^{[m]}\left(\Delta t v_{f}^{i}\right) A_{i},
$$

where $\left(\Delta t v_{f}^{i}\right) A_{i}$ represents the total volume of the advective control volume with positive (negative) inner product of $v_{f}^{i} A_{i}$ representing inflow (outflow), since $A_{i}$ points inwards towards the zone center. Hence equation (2.30) can be interpreted as a net flux of volume through each of the zone faces

$$
\left(F^{[m]}\right)^{n+1}=\left(F^{[m]}\right)^{n}+\frac{1}{V_{Z}} \sum_{Z F} \delta V_{Z F}^{[m]} \frac{v_{f}^{i} A_{i}}{\left|v_{f}^{i} A_{i}\right|+\delta} .
$$

The following sections describe several methods for computing $\delta V_{Z F}$ for an arbitrary number of fluids and fluid distributions on unstructured meshes which satisfy the implicit volume fraction constraints.

However, before going on to describe the interface capturing algorithms, three additional ingredients are introduced here that are needed in all the methods: a renormalization step to enforce the volume fraction constraints, a method to identify the upstream cell, and the calculation of the advection control volume $V_{a c v}$.

The advection control volume is the volume formed by a face of the donor cell and the characteristics projected off the nodes of the face using the local mesh velocity. In $3 \mathrm{D}$, the contribution to the total advection control volume from each of the tetrahedral sub-volume side elements attached to one edgelength of the face is

$$
\begin{aligned}
V_{a c v}^{S} & =\frac{1}{6}\left(\vec{X}_{a c v}-\vec{X}_{F}\right) \cdot\left(\vec{X}_{N 1}-\vec{X}_{F}\right) \times\left(\vec{X}_{N 2}-\vec{X}_{F}\right) \\
& +\frac{1}{6}\left(\vec{X}_{a c v}-\vec{X}_{B}\right) \cdot\left(\vec{X}_{N 2}+\vec{\Delta}_{2}-\vec{X}_{B}\right) \times\left(\vec{X}_{N 1}+\vec{\Delta}_{1}-\vec{X}_{B}\right) \\
& +\frac{1}{6} \sum_{i, j=1}^{2}\left(\vec{X}_{a c v}-\vec{X}_{F \perp}\right) \cdot\left(\vec{X}_{N i}+\vec{\Delta}_{i j}-\vec{X}_{F \perp}\right) \times\left(\vec{X}_{N j}+\vec{\Delta}_{i j}^{*}-\vec{X}_{F \perp}\right)
\end{aligned}
$$

$\vec{X}_{N 1}$ and $\vec{X}_{N 2}$ are the coordinate positions of the two nodes making up a single edgelength of the face or side unit, $\vec{X}_{F}$ is the face center,

$$
\vec{X}_{B}=\frac{1}{N_{T}} \sum_{N=1}^{N_{T}}\left(\vec{X}_{N}+\Delta t \vec{v}_{g, N}\right)
$$

is the center of the back face,

$$
\vec{X}_{a c v}=\frac{1}{2 N_{T}} \sum_{N=1}^{N_{T}}\left(2 \vec{X}_{N}+\Delta t \vec{v}_{g, N}\right)
$$

is the center of the advection control volume, and

$$
\vec{X}_{F \perp}=\frac{1}{4}\left(2 \vec{X}_{N 1}+2 \vec{X}_{N 2}+\vec{\Delta}_{1}+\vec{\Delta}_{2}\right)
$$


is the center of the face formed by the two nodes $N 1$ and $N 2$ and the projected nodal characteristics. Also, $N_{T}$ is the total number of nodes in the face, $\vec{\Delta}_{1}=\Delta t \vec{v}_{g, N 1}, \vec{\Delta}_{2}=\Delta t \vec{v}_{g N 2}$, and $\vec{\Delta}_{i j}$ and $\vec{\Delta}_{i j}^{*}$ are tensors of vectors

$$
\vec{\Delta}_{i j}=\left(\begin{array}{cc}
0 & \vec{\Delta}_{1} \\
0 & \vec{\Delta}_{2}
\end{array}\right), \quad \vec{\Delta}_{i j}^{*}=\left(\begin{array}{cc}
\vec{\Delta}_{1} & \vec{\Delta}_{2} \\
0 & 0
\end{array}\right) .
$$

The total advective control volume is the sum of (2.32) over all edges or side volumes in the face: $V_{a c v}=\sum_{S} V_{a c v}^{S}$.

For a specified donor cell face, the corresponding upstream or opposite face cell is identified by evaluating the intersection of all the other face normals with the effective plane of the target donor cell face. Given a fluxing face with normal $\vec{N}_{0}$ (defined as the sum of the constituent face-faceted side normals) and face-center position $\vec{X}_{F 0}$, construct a vector originating from the face center to an arbitrarily distant position along the face normal: $\vec{X}_{E}=\vec{X}_{F 0}+c \vec{N}_{0}$, where $c=c_{0} V_{Z} /\left(L^{D-1}\right), c_{0}$ is a constant much greater than unity to guarantee the vector $c \vec{N}_{0}$ extends beyond the cell domain, $L$ is a characteristic (minimum) cell length scale, and $D$ is the number of space dimensions. Then loop over each of the other $(j)$ faces in the donor cell with normals $\vec{N}^{(j)}$ and face centers $\vec{X}_{F}^{(j)}$, and evaluate the intersection parameter

$$
T^{(j)}=-\frac{X_{F 0}^{i} N_{i}^{(j)}-X_{F}^{i} N_{i}^{(j)}}{X_{E}^{i} N_{i}^{(j)}-X_{F 0}^{i} N_{i}^{(j)}+\delta} .
$$

The vector $\vec{X}_{E}-\vec{X}_{F 0}$ intersects the plane $\vec{N}^{(j)}$ if $T^{(j)}$ is bounded by $0<T^{(j)}<1$. The upstream cell associated with a specified face is defined as the neighboring zone opposite the donor cell which shares the face with the minimum intersection parameter.

Finally, all the interface advection methods discussed below are supplemented with a volume fraction renormalization step to enforce three additional constraints. First, the volume of fluid fluxed through each of the faces in a donor cell is limited so as not to deplete more material than is available in the cell. Second, to prevent overfluxing into acceptor cells, the outward pointing flux of each material across the faces in each donor cell is conservatively redistributed across all neighboring cells in proportion to either the magnitude of the orthogonal (to the fluxing face) velocity component or the net flux transfer in order to preserve the fluid volume and maintain the constraint $\sum_{m} F^{[m]}=1$. Third, the computed fluxes are conservatively redistributed again, but this time across each of the materials within a single cell and in proportion to $F^{[m]}$ to keep individual fluxes in the range $0 \leq F^{[m]} \leq 1$ while also preserving $\sum_{m} F^{[m]}=1$.

\subsection{Material Ordering Schemes}

An integral part of multi-fluid calculations is the automatic and locally adjustable ordering of all the different materials for advection with little to no user intervention. This effectively allows for an accumulated fluid composition to be formed from materials of relatively higher weighted precedence, thereby filling the donor cell from the downstream to upstream direction, eliminating the need to track interface boundaries on all sides of each material. A single composite volume fraction is defined as

$$
F_{\downarrow}^{[m]}=\min \left[1, \sum_{\ell=1}^{m} F^{[\ell]}\right],
$$


where $F_{\downarrow}^{[m]}$ denotes the sum of volume fractions from the first $m$ ordered materials. The advection process is repeated for each composite group of materials to compute the accumulated transfer volumes $\delta V_{\downarrow}^{[m]}$ across each face of the donor cell. The individual material fluxes are recovered through the normalization

$$
\frac{\delta V^{[m]}}{V_{Z}}=\frac{\max \left[0, \delta V_{\downarrow}^{[m]}-\sum_{\ell=1}^{m-1} \delta V^{[\ell]}\right]}{\sum_{\ell=1}^{M} \delta V^{[\ell]}} \frac{V_{a c v}}{V_{Z}},
$$

where $V_{a c v}$ is the advection control volume and $\delta V^{[m]}$ is the $m$ th fluid material transfer volume bounded by the interface surface and the advection control volume. The normalization is designed to limit the total volume fraction from exceeding unity, and the individual volume fractions from becoming negative due to numerical errors.

Two ordering schemes have been implemented: downstream- and upstream-loaded, distinguished by the relative volume weights of the fluid distribution. In the first scheme, materials are ordered into four distinctly defined groups plus an additional fifth group to accomodate materials not falling into the other four. The first group is the highest priority in the advection process since it stores the predominantly downstream materials. Using the notation $F_{D-1}^{[m]}, F_{D}^{[m]}$ and $F_{D+1}^{[m]}$ to denote the upstream, donor and downstream cells for material $m$ respectively, fluids falling into this category satisfy $F_{D-1}^{[m]}<F_{c},\left(F_{D}^{[m]}, F_{D+1}^{[m]}\right)>F_{c}$, and represent a surface that is essentially parallel to the cell face orientation. Note that $F_{D-1}^{[m]}$ can be assigned as the volume fraction in the upstream cell using the identification process described above, or simply by

$$
F_{D-1}^{[m]}=F_{D+1}^{[m]}-2\left(\nabla_{i} F\right)_{Z}^{L}\left(X_{A}^{i}-X_{Z}^{i}\right)
$$

where $\left(\nabla_{i} F\right)_{Z}^{L}$ is the donor cell-centered limited gradient of the volume fraction, and $X_{A}^{i}$ and $X_{Z}^{i}$ are the acceptor and donor cell centers respectively. The second group includes materials with surfaces aligned more perpendicular to the cell face (or more parallel to the face normal) and are characterized by $\left(F_{D-1}^{[m]}, F_{D}^{[m]}, F_{D+1}^{[m]}\right)>F_{c}$. The third group represents isolated fragments with $\left(F_{D-1}^{[m]}, F_{D+1}^{[m]}\right)<F_{c}$ and $F_{D}^{[m]}>F_{c}$ which are advected prior to the dominantly upstream or trailing materials characterized by $F_{D+1}^{[m]}<F_{c}$ and $\left(F_{D-1}^{[m]}, F_{D}^{[m]}\right)>F_{c}$. However, it is possible that more than one material can fall into each category, making it necessary to implement a more quantitative procedure to order the fluids within each group. Since the general idea is to advect from leading to trailing edge materials, a simple calculation of the normalized volume fraction gradient provides a convenient measure of priority. For the first, second and fourth groups, the order parameter is defined as $P=\left(F_{D+1}-F_{D-1}\right) / F_{D}$. This parameter naturally increases (decreases) for higher volume fractions in the downstream (upstream) cells. It also increases in magnitude for smaller donor cell fractions when the upstream or downstream cells are saturated to unit fraction levels, reflecting the "steeper" nature of the interface surface. For the third group in which there is little or no fluid present in either the upstream or downstream cells, the order parameter is simply the volume fraction in the donor cell $P=F_{D}$.

The second (predominantly upstream weighted) ordering scheme is essentially the mirror image of the first, but with some minor adjustments. In particular, the highest priority group in this ordering scheme includes the isolated fragments satisfying $\left(F_{D-1}^{[m]}, F_{D+1}^{[m]}\right)<F_{c}$ and $F_{D}^{[m]}>F_{c}$ with priority $P=1 / F_{D}$. The other groups are ordered according to the relative presence of materials 
in the upstream cell: $F_{D-1}^{[m]}<F_{c}, F_{D}^{[m]}>F_{c}, F_{D+1}^{[m]}>1-F_{c}$ with priority $P=1 / F_{D}$ for the second group, $F_{D-1}^{[m]}<F_{c}, F_{D}^{[m]}>F_{c}, F_{D+1}^{[m]}>F_{D}$ with priority $P=F_{D+1} / F_{D}$ for the third group, and $F_{D-1}^{[m]}>F_{c}, F_{D}^{[m]}>F_{c}, F_{D+1}^{[m]}>F_{c}$ with priority $P=\left(F_{D+1}-F_{D-1}\right) / F_{D}$ for the fourth. This second scheme can be effective in some advection algorithms that are more prone to generating floating debris. These ordering schemes are applied as a first step in all interface capturing algorithms discussed below.

\subsection{Aligned Bisection Method}

Some of the earliest directionally split VoF algorithms (DeBar 1974; Noh \& Woodward 1976; Hirt \& Nichols 1981) assume the interface surface is aligned either parallel or perpendicular to the flow vector component in each dimensional sweep. These algorithms thus work well if the flow geometry is primarily aligned parallel to one coordinate direction or along the velocity vector field. However, they can become highly inaccurate for more complex geometries or off-axis translational and rotational flows.

By generalizing these algorithms to allow for "corners" inside a single cell, it is possible to more accurately represent arbitrary surface orientations with several adjustable bricks of fluid within the donor cell, effectively increasing the curvature modeling capabilities at smaller scales (Anninos 2000). A modified aligned algorithm with improved accuracy and generalized to unsplit advection is achieved by introducing four (six) parameters in 2D (3D) representing the dimensions of two distinct blocks of fluid which run the length of the donor cell along the face normals for each face of the cell. Assuming a face-normal vector pointing in the $x$ direction, the width and height dimensions of the two fluid blocks are denoted by $\delta x_{1}, \delta x_{2}, \delta y_{1}, \delta y_{2}, \delta z_{1}$, and $\delta z_{2}$ with the block labeled with subscripts 1 (2) being more downstream (upstream) than the other. The normalized height functions are associated with volume fractions in the neighboring downstream and upstream cells

$$
\frac{\delta y_{1}}{\Delta y}=\frac{\delta z_{1}}{\Delta z}=\sqrt{F_{D+1}}
$$

and

$$
\frac{\delta y_{2}}{\Delta y}=\frac{\delta z_{2}}{\Delta z}=\sqrt{F_{D-1}}
$$

where $\Delta y$ and $\Delta z$ are the characteristic transverse cell dimensions, and where the filling factors for each of the cross sectional areas have been assumed to be equal and related to the appropriate adjoining cell volume fraction. The width parameters are determined by the constraints

$$
\frac{\delta x_{1}}{\Delta x}+\frac{\delta x_{2}}{\Delta x}=1
$$

and

$$
\delta x_{1} \delta y_{1} \delta z_{1}+\delta x_{2} \delta y_{2} \delta z_{2}=F_{D} V_{Z} .
$$

The fluid flux is then defined as

$$
\frac{\delta V}{V_{a c v}}=F_{D+1}
$$

if $\left(V_{a c v} / V_{Z}\right) \leq\left(\delta x_{1} / \Delta x\right)$, or

$$
\frac{\delta V}{V_{a c v}}=\frac{V_{Z}}{V_{a c v}} \frac{\delta x_{1}}{\Delta x} F_{D+1}+\left(1-\frac{\delta x_{1}}{\Delta x} \frac{V_{Z}}{V_{a c v}}\right) F_{D-1}
$$


otherwise, and where

$$
\frac{\delta x_{1}}{\Delta x}=\frac{\left(F_{D}-F_{D-1}\right)}{F_{D+1}-F_{D-1}} .
$$

For cases in which $\delta x_{1} / \Delta x \leq 0$, the transfer volume is set to $\delta V=F_{D} V_{a c v}$, and if $\delta x_{1} / \Delta x \geq$ $0, \delta V=F_{D+1} V_{a c v}$. Notice that this method does not require explicit knowledge of the precise orientation of the material interface: the compound block structure is aligned relative to the cell face normals and planes, and sized in dimensions to satisfy the volume fraction constraints.

\subsection{Donor/Acceptor Limiter}

An alternative, but slightly more computationally expensive, method to that described in $\S 2.2$ is the donor/acceptor concept which is based on switching between the donor or downstream cell volume fractions to flatten or steepen the fluid distribution as appropriate. A fairly robust algorithm can be developed using minimal cell connectivity data and extended to unsplit advection. For each face, it is necessary only to estimate the interface orientation or slope $(s)$ from the local volume fraction gradients, to define a reference vector against which the interface normal is compared, to identify the appropriate donor or downstream volume fractions for each outflow cell face, and to apply the necessary volume constraint preserving steps.

This approach utilizes a parameterized flux limiting formula to compute the volume of advected fluid using a downstream filling algorithm that is aligned relative to the face normal orientation. The flux limiter can be conveniently written in compact form as

$$
\frac{\delta V}{V_{a c v}}=\min \left[\eta+\max \left((1-\eta)-\left(1-\tilde{F}_{D}\right) \frac{V_{Z}}{V_{a c v}}, 0\right), \tilde{F}_{D} \frac{V_{Z}}{V_{a c v}}\right]
$$

where

$$
\tilde{F}_{D}=F_{D}+\frac{1}{2} \frac{d F}{d V}\left(V_{Z}-V_{a . c v}\right)
$$

$\eta=F_{D+1}$ if the slope $s>s_{c}$ (with a critical slope $s_{c}$ typically set to one or two), or $\eta=\tilde{F}_{D}$ otherwise. Expression (2.48) is derived by considering the various independent orthogonal fluid distributions in the donor and acceptor cells. The volume fraction derivative is defined as

$$
\frac{d F}{d V}=\max \left(\frac{2\left(F_{D+1}-F_{D}\right)}{V_{D}+V_{A}}, \frac{2\left(F_{D}-F_{D-1}\right)}{V_{D}+V_{U}}\right)
$$

where $V_{D} \equiv V_{Z}, V_{U}$, and $V_{A}$ are the donor, upstream and acceptor cell volumes. To force a steeper boundary and help prevent the generation of floating debris, the transfer volume is redefined with $\eta=F_{D+1}$ if $F_{D+1}<F_{c}, F_{D} \geq F_{c}$ and $F_{D-1}>1-F_{c}$, or if $F_{D-1}<F_{c}$ and $F_{D} \geq F_{c}$.

There are many ways to estimate the slope of the interface boundary. The most straightforward procedure is to associate the three-dimensional surface parameters with the local volume fraction gradients projected to each of the face normals separately. First, compute the volume fraction gradients using the most compressive (superbee) limited gradient operator defined in $\S 1.1$ and split the gradients into perpendicular

$$
\left(\nabla_{i} F\right)_{\perp}^{L}=\left(\nabla_{j} F\right)_{Z}^{L} \frac{N_{F}^{j} N_{F}^{i}}{N_{F}^{j} N_{j}^{F}},
$$


and parallel

$$
\left(\nabla_{i} F\right)_{\|}^{L}=\left(\nabla_{i} F\right)_{Z}^{L}-\left(\nabla_{i} F\right)_{\perp}^{L}
$$

components relative to the advecting cell face. $N_{F}^{i}$ is a vector normal to the advecting cell face, and defined as the sum of the face-faceted area vectors of the tetrahedral side elements within the face. The interface slope is then defined as

$$
s=\frac{\left|F_{D+1}-F_{D}\right|}{\Delta X_{A D}\left|(\nabla F)_{\|}^{L}\right|+\delta} \max (0, \max (\min (1,2 \theta), \min (2, \theta))),
$$

where $X_{A D}$ is the distance between donor and acceptor cells, and

$$
\theta=\frac{F_{D}-F_{D-1}}{F_{D+1}-F_{D}+\delta}
$$

to force an additional monotonicity of the volume fraction variance orthogonal to the face.

\subsection{Series/Parallel Models}

The final group of methods discussed here are similar to the donor/acceptor method in $\S 2.3$ in that they all use the interface slope (as defined in \$2.3) to switch predominantly between two different fluid topologies: series or steep $\left(s>s_{c}\right)$ versus parallel or flat $\left(s \leq s_{c}\right)$ flow with $s_{c}=1$ (Tipton 1994). Materials that have been grouped as series are given the highest (lowest) priority in the advection sequence if the material is dominantly downstream (upstream) as described in section 2.1. The volume flux for this group of materials is computed in a grid aligned manner, and assigned as

$$
\frac{\delta V}{V_{a c v}}=\min \left(1, F_{D} \frac{V_{Z}}{V_{a c v}}\right),
$$

for leading and middle materials classified as series, or

$$
\frac{\delta V}{V_{a c v}}=\min \left(1, \max \left(0,1-\left(1-F_{D}\right) \frac{V_{Z}}{V_{a c v}}\right)\right),
$$

for trailing materials classified as series. Fluids grouped as parallel are given intermediate priority with transfer volumes:

$$
\frac{\delta V}{V_{a c v}}=\min \left(1, \max \left(0, \min \left(\frac{F_{D} V_{Z}}{V_{a c v}}, \bar{F}\right)\right)\right)
$$

where

$$
\bar{F}=F_{D}+\left(V_{Z}-V_{a c v}\right) \max \left(\frac{F_{D+1}-F_{D}}{V_{Z}+V_{A}}, \frac{F_{D}-F_{D-1}}{V_{Z}+V_{U}}\right)+c X_{A D}^{i}\left(\nabla_{i} F\right)_{\|}^{L},
$$

where $c$ is a constant to include $(c=1)$ or not $(c=0)$ the transverse interpolant.

Other topological parameters, in addition to the slope, can be used to force a classification of the material distribution as either series or parallel in ambiguous situations. For example, the flow is considered as series if either $F_{D+1}<\delta$ and $F_{D}>0.1$, or $F_{D}>(1+\delta) F_{D-1}$, or $F_{D-1}<\delta$ and $F_{D+1}>1-\delta$, or $F_{D+1}<\delta$ and $F_{D-1}>1-\delta$, where $\delta \ll 1$. The transfer volume can also be set to

$$
\frac{\delta V}{V_{a c v}}=\min \left(1, \frac{F_{D+1} V_{Z}}{V_{a c v}}\right)
$$

if $F_{D-1}<\delta$ and $F_{D+1}>1-\delta$. 


\subsection{Advection with volume fractions}

The conservative first stage of the remap cycle, equation (1.5), can be rewritten to explicitly include the volume fraction in the mass and energy conservation equations as

$$
\begin{aligned}
\frac{\partial\left(F^{[m]} \rho^{[m]}\right)}{\partial t} & =\nabla_{i}\left(F^{[m]} \rho^{[m]} v_{g}^{i}\right), \\
\frac{\partial\left(\rho v^{k}\right)}{\partial t} & =\nabla_{i}\left(\rho v^{k} v_{g}^{i}\right), \\
\frac{\partial\left(F^{[m]} \rho^{[m]} \epsilon^{[m]}\right)}{\partial t} & =\nabla_{i}\left(F^{[m]} \rho^{[m]} \epsilon^{[m]} v_{g}^{i}\right),
\end{aligned}
$$

with the following constraints:

$$
\sum_{m} F^{[m]}=1, \quad \sum_{m} F^{[m]} \rho^{[m]}=\rho, \quad \sum_{m} F^{[m]} \rho^{[m]} \epsilon^{[m]}=\rho \epsilon
$$

The momentum equation update is not affected beyond the basic approach outlined in $\S 1.2$ since the total volume fraction weighted density is used in (2.61) and the velocity is common to all the materials. The mass density and energy equations (2.60) and (2.62) are discretized with finite volumes and solved for each material as

$$
\begin{aligned}
F^{[m], n+1 / 2} \rho^{[m], n+1 / 2} & =F^{[m], n} \rho^{[m], n}-\frac{1}{V_{Z}^{n}} \sum_{F} \delta V_{F}^{[m], n} \rho_{* F}^{[m], n} \frac{v_{g}^{i} A_{i}}{\left|v_{g}^{i} A_{i}\right|+\delta} \\
F^{[m], n+1 / 2} \rho^{[m], n+1 / 2} \epsilon^{[m], n+1 / 2} & =F^{[m], n} \rho^{[m], n} \epsilon^{[m], n}-\frac{1}{V_{Z}^{n}} \sum_{F} \delta V_{F}^{[m], n} \rho_{* F}^{[m], n} \epsilon_{* F}^{[m], n} \frac{v_{g}^{i} A_{i}}{\left|v_{g}^{i} A_{i}\right|+\delta^{2 .}}
\end{aligned}
$$

after $F^{[m], n+1 / 2}$ is computed by

$$
F^{[m], n+1 / 2}=F^{[m], n}-\frac{1}{V_{Z}^{n}} \sum_{Z F} \delta V_{Z F}^{[m], n} \frac{v_{g}^{i} A_{i}}{\left|v_{g}^{i} A_{i}\right|+\delta} .
$$

Here $\rho_{* F}^{[m], n}$ and $\epsilon_{* F}^{[m], n}$ are the upwind values of the density and specific energy for material $[m]$, at time level $n$, upwind from face $F$, and either centered in the donor cell or extrapolated to the face or advection control volume centers using a first order monotonic expansion as in (1.12) if the extrapolation is not performed across an interface. The remap cycle is completed to time level $n$ by applying the operator split compression contribution (1.6) to each of the intermediate solutions (2.64) - (2.66).

\section{Multiphase 2T RHD with material interfaces}

\subsection{Multiphase model}

Expanding equations (2.60) - (2.62) to include the compressive remap term and the physical Lagrangian elements, the multiphase continuity equations coupled with a $2 \mathrm{~T}$ radiation diffusion model 
can be written in ALE form as

$$
\begin{aligned}
\frac{\partial\left(F^{[m]} \rho^{[m]}\right)}{\partial t}= & -\nabla_{i}\left(F^{[m]} \rho^{[m]}\left(v^{i}-v_{g}^{i}\right)\right)-F^{[m]} \rho^{[m]} \nabla_{i} v_{g}^{i}, \\
\frac{\partial\left(\rho v^{k}\right)}{\partial t}= & -\nabla_{i}\left(\rho v^{k}\left(v^{i}-v_{g}^{i}\right)\right)-\rho v^{k} \nabla_{i} v_{g}^{i}-\nabla_{k}\left(P+P_{R}\right), \\
\frac{\partial\left(F^{[m]} \rho^{[m]} \epsilon^{[m]}\right)}{\partial t}= & -\nabla_{i}\left(F^{[m]} \rho^{[m]} \epsilon^{[m]}\left(v^{i}-v_{g}^{i}\right)\right)-F^{[m]} \rho^{[m]} \epsilon^{[m]} \nabla_{i} v_{g}^{i} \\
& -h^{[m]} P^{[m]} \nabla_{i} v^{i}+g^{[m]}\left(\sigma E-\sigma a T_{e}^{4}\right), \\
\frac{1}{c} \frac{\partial E}{\partial t}= & -\nabla_{i}\left(E\left(v^{i}-v_{g}^{i}\right)\right)-E \nabla_{i} v_{g}^{i}+\nabla_{i}\left(D \nabla^{i} E-\frac{4}{3} E v^{i}\right)-\sigma E+\sigma a T_{e}^{4},
\end{aligned}
$$

where $v^{k}$ is the physical velocity assumed to be the same for each material, $v_{g}^{k}$ the grid velocity, $\rho=$ $\sum_{m} F^{[m]} \rho^{[m]}$ the average density, $\rho \epsilon=\sum_{m} F^{[m]} \rho^{[m]} \epsilon^{[m]}$ the average energy density, $P$ the effective material pressure in a mixed cell, $P_{R}=E / 3$ the radiation pressure, $E$ the homogeneous (single material) radiation energy, $D$ the diffusion coefficient, $\sigma$ the crossection, $T_{e}$ the effective single material electron temperature, $g^{[m]}$ is an energy weight function for distributing the homogeneous (across materials) radiation energy into each material, and $h^{[m]}$ and $P^{[m]}$ in the energy equation (3.69) are the weighting coefficient for compression work and pressure for each material $[m]$. The compression weight factor is taken to be the normalized adiabatic compressibility or bulk modulus $K_{S}=\rho \partial P /\left.\partial \rho\right|_{S}$ at constant entropy (Youngs 1994)

$$
h^{[m]}=\frac{F^{[m]} \bar{K}_{S}}{K_{S}^{[m]}},
$$

with average bulk modulus

$$
\bar{K}_{S}=\frac{1}{\sum_{m} F^{[m]} / K_{S}^{[m]}},
$$

defined such that $\sum_{m} h^{[m]}=1$. The effective pressure is set either as the sum of the partial pressures $\left(P=\sum_{m} P^{[m]}\right)$ if the multiphase model is not used, or the bulk modulus weighted average

$$
P \equiv \bar{P}=\sum_{m} \frac{P^{[m]} F^{[m]} \bar{K}_{S}}{K_{S}^{[m]}}
$$

otherwise.

In the multiphase model, the component pressures within a single cell can be adjusted as an option to drive the mix to pressure equilibrium at the common pressure (3.73). This is achieved by altering the volumes which each material occupies in a cell to the form

$$
\delta P^{[m]}=\bar{P}-P^{[m]}=-K_{S}^{[m]} \frac{\delta V^{[m]}}{V^{[m]}} .
$$

This amounts to changing the local volume fractions as

$$
\frac{\delta F^{[m]}}{F^{[m]}}=\frac{P^{[m]}-\bar{P}}{K_{S}^{[m]}}+\frac{\delta V_{Z}}{V_{Z}}\left(\frac{\bar{K}_{S}}{K_{S}^{[m]}}-1\right) .
$$


In practice, (3.75) is limited to maximum fractional changes $|\delta F / F|_{\max } \leq 0.1$, and over time scales linked to the local sound speed: $\delta F^{[m]} \rightarrow k_{C s} \delta F^{[m]}$, where $k_{C s}=0.5 \min \left(1, \Delta t \min \left(C_{s}\right) / \Delta l\right)$, and $\Delta t$ is the timestep, $\Delta l$ is a charateristic minimum length scale, and $\min \left(C_{s}\right)$ is the smallest sound speed over all materials in the cell. Substituting $\left(K_{S}^{[m]} F^{[m]} / \rho^{[m]}\right)^{1 / 2}$ for the material sound speed, the volume fraction is adjusted according to

$$
\delta F^{[m]}=\min \left(\left|\frac{\delta F}{F}\right|_{\max }\left|\frac{\delta \tilde{F}^{[m]}}{\tilde{F}^{[m]}}\right|^{-1}\left|\delta \tilde{F}^{[m]}\right|,\left|\delta \tilde{F}^{[m]}\right|\right) \frac{\delta \tilde{F}^{[m]}}{\left|\delta \tilde{F}^{[m]}\right|}
$$

where

$\delta \tilde{F}^{[m]}=k \min \left(1, \frac{\Delta t}{\Delta l} \min \left(\sqrt{\frac{K_{S}^{[m]} F^{[m]}}{\rho^{[m]}}}\right)\right)\left(\frac{\left(P^{[m]}-\bar{P}\right) F^{[m]}}{K_{S}^{[m]}}+\left(\frac{\bar{K}_{S}}{K_{S}^{[m]}}-1\right) \frac{2 F^{[m]}\left(V_{Z}^{n+1}-V_{Z}^{n}\right)}{V_{Z}^{n+1}+V_{Z}^{n}}\right)$,

and the constant $k<1$ is typically set to $1 / 2$. Each of the volume fraction corrections $\delta F^{[m]}$ performed in a cell are subsequently renormalized to enforce the constraints $\sum_{m} \delta F^{[m]}=0$ and $0 \leq F^{[m]} \leq 1$. After renormalization, the specific internal energy is adjusted to account for the new thermodynamic state

$$
\delta \epsilon^{[m]}=-\frac{P^{[m]}}{\rho^{[m]}} \frac{\delta V^{[m]}}{V^{[m]}}=-\frac{P^{[m]}}{\rho^{[m]}} \frac{\delta F^{[m]}}{F^{[m]}}
$$

\subsection{Radiation diffusion with material interfaces}

The coupling between radiation and hydrodynamics is achieved by assuming a constant specific heat over the solve cycle and rewriting (3.69) as

$$
F^{[m]} \rho^{[m]} C_{v}^{[m]} \frac{\partial T^{[m]}}{\partial t}=-F^{[m]} \rho^{[m]} \nabla_{i} C_{v}^{[m]} T^{[m]}\left(v^{i}-v_{g}^{i}\right)-h^{[m]} P^{[m]} \nabla_{i} v^{i}+F^{[m]} \sigma^{[m]}\left(E-a T_{e}^{4}\right)
$$

using the individual material cross sections. An effective single material energy equation is constructed by defining

$$
T=\frac{\sum F^{[m]} \rho^{[m]} C_{v}^{[m]} T^{[m]}}{\sum F^{[m]} \rho^{[m]} C_{v}^{[m]}}, \quad C_{v}=\frac{\sum F^{[m]} \rho^{[m]} C_{v}^{[m]}}{\sum F^{[m]} \rho^{[m]}}, \quad \text { and } \quad \sigma=\sum F^{[m]} \sigma^{[m]}
$$

to write the non-convective terms in (3.79) as

$$
\begin{aligned}
\frac{\rho C_{V}}{4 T_{e}^{3}} \frac{\partial T_{4 e}}{\partial t} & =-Q_{p d v}+a \sigma\left(T_{4 R}-T_{4 e}\right), \\
\frac{1}{c} \frac{\partial E}{\partial t} & =\nabla_{i}\left(D \nabla^{i} E-\frac{4}{3} E v^{i}\right)-\sigma\left(E-a T_{4 e}\right),
\end{aligned}
$$

where the notation $T_{4} \equiv T^{4}$ is introduced for both the radiation $\left(T_{4 R}\right)$ and electron $\left(T_{4 e}\right)$ temperatures. Also, $E=a T_{R}^{4}$, and

$$
Q_{p d v}=\sum_{m} Q_{p d v}^{[m]}=\sum_{m} h^{[m]} p^{[m]} \nabla_{i} v^{i}=P \nabla_{i} v^{i}
$$


Three procedures are described below that can be used to easily extend single material radiation diffusion solvers to include multi-material interfaces. These procedures are based on formulating reasonable choices for the weight coefficient $g^{[m]}$ in equation (3.69), given a solution to the homogenous diffusion equation (3.82).

Considering only the non-convective terms, the first method constructs a first order semi-implicit solution to the discretized multiphase internal energy equations using the time advanced solution $\left(T_{4 R}^{n+1}\right)$ to the diffusion equation

$$
\frac{F^{[m]} \rho^{[m]} C_{v}^{[m]}}{4\left(T_{*}^{[m]}\right)^{3} \Delta t}\left(T_{4 e}^{[m], n+1}-T_{4 e}^{[m], n}\right)=-Q_{p d v}^{[m]}+a F^{[m]} \sigma^{[m]}\left(T_{4 R}^{n+1}-T_{4 e}^{[m], n+1}\right) .
$$

Equation (3.84) can be solved algebraically for $T_{4 e}^{[m], n+1}$

$$
T_{4 e}^{[m], n+1}=\frac{F^{[m]} \rho^{[m]} C_{v}^{[m]} T_{4 e}^{[m], n}+4\left(T_{*}^{[m]}\right)^{3}\left(a \Delta t^{[m]} F^{[m]} \sigma^{[m]} T_{4 R}^{n+1}-\Delta t Q_{p d v}^{[m]}\right)}{F^{[m]} \rho^{[m]} C_{v}^{[m]}+4 a \Delta t^{[m]} F^{[m]} \sigma^{[m]}\left(T_{*}^{[m]}\right)^{3}}
$$

where $T_{*}^{[m]}$ is a reference temperature about which the solution is linearized and is chosen as either the time-retarded, time-advanced, or time-averaged electron temperature. Also,

$$
\Delta t^{[m]}=\frac{k_{C R} T_{4 e}^{n} F^{[m]} \rho^{[m]} C_{v}^{[m]}}{4\left(T_{*}^{[m]}\right)^{3}\left(Q_{p d v}^{[m]}+a F^{[m]} \sigma^{[m]}\left(T_{4 R}^{n+1}-T_{4 e}^{[m], n}\right)\right)}
$$

is a pseudo-timestep defined with constant coefficient $k_{C R}<1$ associated with the physical radiation Courant factor to keep temperature variations for each material relatively small.

A second method follows the basic idea of the first, except that weighted combinations of variables are used to make results at extremely low volume fractions more robust and constrained to relative low scatter. Temperatures in this method follow (3.85)

$$
T_{4}^{[m], n+1}=\frac{F^{[m]} \rho^{[m]} C_{v}^{[m]} T_{* 4}^{[m]}+4\left(T_{*}^{[m]}\right)^{3}\left(a \Delta t^{[m]} F^{[m]} \sigma^{[m]} T_{4 R}^{n+1}-\Delta t \tilde{Q}_{p d v}^{[m]}\right)}{F^{[m]} \rho^{[m]} C_{v}^{[m]}+4 a \Delta t^{[m]} F^{[m]} \sigma^{[m]}\left(T_{*}^{[m]}\right)^{3}}
$$

with

$$
\begin{aligned}
& \tilde{Q}_{p d v}^{[m]}=F^{[m]} Q_{p d v}^{[m]}+\left(1-F^{[m]}\right) \sum_{j} F^{[j]} Q_{p d v}^{[j]}, \\
& T_{*}^{[m]}=F^{[m]} T_{e}^{[m], n}+\left(1-F^{[m]}\right) \sum_{j} F^{[j]} T_{e}^{[j], n},
\end{aligned}
$$

and

$$
\Delta t^{[m]}=\frac{k_{C R} T_{* 4}^{[m]} F^{[m]} \rho^{[m]} C_{v}^{[m]}}{4\left(T_{*}^{[m]}\right)^{3}\left(\tilde{Q}_{p d v}^{[m]}+a F^{[m]} \sigma^{[m]}\left(T_{4 R}^{n+1}-T_{* 4}^{[m], n}\right)\right.} .
$$

The third and final procedure presented here defines the temperature simply as a specific heat weighted average of all materials within the cell

$$
T^{[m], n+1}=T^{[m], n}+\frac{\lambda^{[m]} \Delta E_{R}-\Delta t Q_{p d v}^{[m]}}{F^{[m]} \rho^{[m]} C_{v}^{[m]}}
$$


where $\Delta E_{R} \equiv \sigma\left(E-a T_{4 e}\right)$ is the total energy change due to radiation effects, and

$$
\lambda^{[m]} \equiv g^{m]}=\frac{F^{[m]} \rho^{[m]} C_{v}^{[m]}}{\sum_{j} F^{[j]} \rho^{[j]} C_{v}^{[j]}}
$$

if $\Delta E_{R}>0$, or

$$
\lambda^{[m]}=\frac{F^{[m]} \rho^{[m]} C_{v}^{[m]} T^{[m]}}{\sum_{j} F^{[j]} \rho^{[j]} C_{v}^{[j]} T^{[j]}}
$$

otherwise. This approach generally yields comparable results to the previous two methods, though it can be less robust for very low volume fraction material, particularly when actual densities are used in place of cell average densities.

In order to guarantee energy conservation at the end of the radiation update cycle, the material temperatures are rescaled after each of the above redistribution calculations according to

$$
T_{e}^{[m], n+1} \rightarrow \alpha T_{e}^{[m], n+1}=\left(\frac{\Delta e+\sum_{j} F^{[j]} \rho^{[j]} C_{v}^{[j]} T_{e}^{[j], n}}{\sum_{j} F^{[j]} \rho^{[j]} C_{v}^{[j]} T_{e}^{[j], n+1}}\right) T_{e}^{[m], n+1}
$$

where $\Delta e$ is the net (radiation $+\mathrm{PdV}$ summed over all materials) change in internal energy density, and the normalization factor $\alpha$ is constructed so that when the internal specific energy is updated by

$$
\epsilon^{[m], n+1}=\epsilon^{[m], n}+C_{v}^{[m]}\left(T_{e}^{[m], n+1}-T_{e}^{[m], n}\right)
$$

the process is conservative over the cycle such that

$$
\sum_{m} F^{[m]} \rho^{[m]} C_{v}^{[m]}\left(T_{e}^{[m], n+1}-T_{e}^{[m], n}\right)=\Delta e=\Delta E_{R}-\Delta t \sum_{m} Q_{p d v}^{[m]}
$$

Acknowledgements This work was performed under the auspices of the U.S. Department of Energy by University of California, Lawrence Livermore National Laboratory under Contract W7405-Eng-48.

\section{References}

[1] R. DeBar, Technical Report UCID-19683, Lawrence Livermore National Laboratory (1974)

[2] W.F. Noh and P.R. Woodward, In Lecture Notes in Physics; 59, A.I. van der Vooren and P.J. Zandbergen, editors, pages 330-340 (Springer-Verlag, 1976)

[3] C.W. Hirt and B.D. Nichols, J. Comput. Phys. 39, 201 (1981)

[4] R. Tipton, Technical Report, Lawrence Livermore National Laboratory (1994)

[5] P. Anninos, Technical Report, Lawrence Livermore National Laboratory (2000)

[6] D.L. Youngs, Laser 8 Particle Beams 12, 725 (1994) 